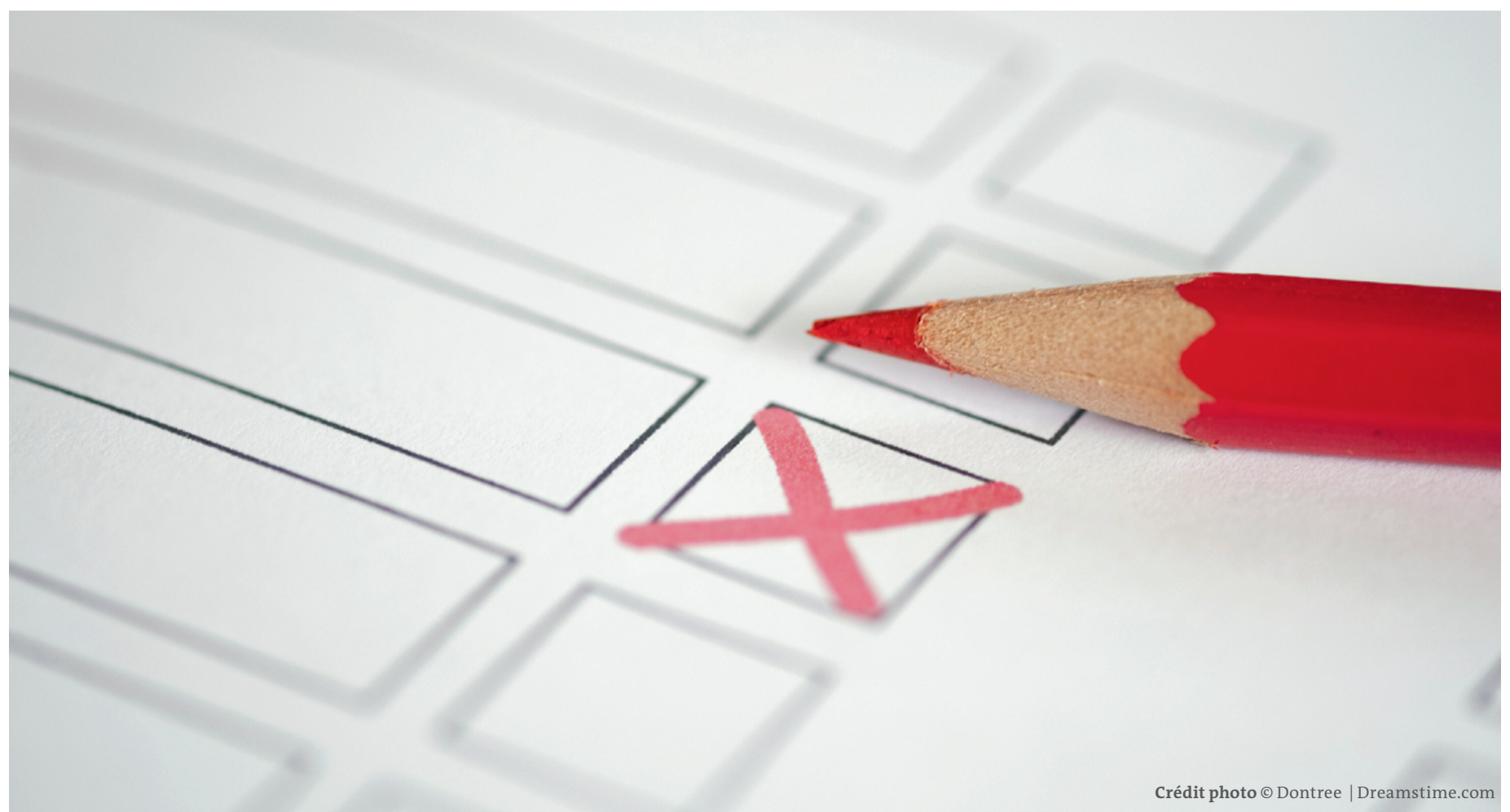

\title{
Elections générales 2020: portraits des candidates et candidats
}

Lors de la Chambre médicale du 7 mai 2020, les déléguées et les délégués sont appelés à renouveler l'ensemble des organes de la FMH pour la législature 20202024. Les candidat-e-s pour les postes du Comité central, de la Commission de gestion et la présidence et vice-présidence de la Commission de déontologie, connus à la clôture de la rédaction, saisissent l'occasion de se présenter en publiant leurs portraits ci-après.

\section{Qui sera élu à la Chambre médicale?}

Lors de la Chambre médicale du 7 mai 2020, il s'agira d'élire les sept membres du Comité central de la $\mathrm{FMH}$, dont la présidente ou le président et deux vice-président-e-s, la présidente ou le président de l'ISFM, les cinq membres de la Commission de gestion, la présidente ou le président et les quatre vice-président-e-s de la Commission de déontologie et les 33 membres de l'Assemblée des délégués. Pour être éligibles, les candidat-e-s doivent notamment être médecins et membres de la FMH. Pour les personnes qui se présentent au Comité central, la Chambre médicale exige également qu'elles remplissent les conditions d'un profil de compétences. 


\section{Candidature: Comité central}

\section{Christoph Bosshard sortant}

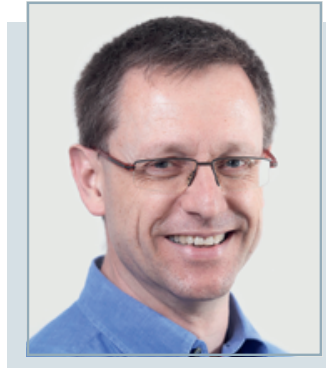

Titre: Dr méd.

Date de naissance: 7.11.1964

Domicile: Berne

Lieu d'origine:Turbenthal

Etat civil: marié, 4 enfants

Courriel: christoph.bosshard@fmh.ch

Titre de spécialiste: chirurgie orthopédique et trauma-

tologie de I'appareil locomoteur

\section{Affiliation à des sociétés médicales}

- Depuis 2014: Swiss Insurance Medicine (SIM)

- Depuis 2009: Société bernoise des chirurgiens orthopédiques

- Depuis 2003: Société suisse d'orthopédie et de traumatologie (swiss orthopaedics)

- Depuis 1993: FMH

- Depuis 1992: Association suisse des médecins assistant(e)s et chef(fe)s de clinique (ASMAC)

\section{Liens d'intérêts}

Médecin dirigeant en médecine des assurances à la Suva

\section{Autres qualifications}

- Depuis 2014: activité d'enseignement en médecine des assurances à l'Université de Berne

- 2011: expert médical certifié SIM

\section{Langues}

Allemand (langue maternelle), français (B2), anglais (B2), italien (A2)

\section{Activité professionnelle actuelle}

- Depuis 2017: médecin dirigeant en médecine des assurances à la Suva

- Depuis 2012: membre du Comité central et vice-président de la FMH, responsable du département Données, démographie et qualité

\section{Parti politique}

aucun

\section{Mandats politiques exercés jusqu'à présent}

aucun

\section{Mandats de politique professionnelle}

- Depuis 2015: vice-président de la FMH

- Depuis 2012: membre du Comité central de la FMH, responsable du département Données, démographie et qualité
- 2008-2012: président de l'Association suisse des médecins assistant(e)s et chef(fe)s de clinique (ASMAC) Suisse

- 2005-2010: président de l'Association suisse des médecins assistant(e)s et chef(fe)s de clinique (ASMAC), section bernoise

- Commission de déontologie de swiss orthopaedics

- Groupe d'experts en médecine des assurances de swiss orthopaedics

- Vice-président de la Société bernoise des chirurgiens orthopédiques

\section{Expérience de direction}

- 2010-2017: responsable du Groupe médical Suva Berne/Fribourg/Sion/Soleure comptant 28 collaborateurs en tout, répartis sur les 4 sites mentionnés

- 2008-2009: médecin d'arrondissement Suva Berne

- 2001-2008: activité de médecin-cadre dans les cliniques orthopédiques du réseau hospitalier Spital Netz Bern, y c. tâches spéciales

- Armée: commandant d'unité; médecin de bataillon

- Cursus de formation pour cadres de la Suva

- Politique professionnelle, voir ci-dessus

\section{Compétences, motivation et objectifs}

Après deux mandats au Comité central, je me mets à disposition de la FMH pour un troisième mandat dans le but de poursuivre le travail déjà accompli sur des sujets cruciaux pour le corps médical et pour le système de santé. Par exemple: la mise en œuvre judicieuse de la nouvelle loi sur la qualité ou la collecte et l'utilisation des données (avec un avantage réel pour le corps médical par rapport au temps investi par les médecins) tout en veillant au respect du cadre légal. Et il s'agit aussi de répondre à l'accroissement des tâches administratives par des moyens innovants. Il reste également de nombreuses tâches à accomplir en interne: unir nos forces et favoriser la mise en réseau dans tous les domaines pour éviter les doublons, et piloter la rénovation du bâtiment de la FMH décidée par la Chambre médicale ne sont que deux autres exemples. Or, ce n'est qu'ensemble que ces tâches peuvent être menées à bien, avec vous, chères et chers collègues. Je vous remercie de votre soutien! 


\section{Candidature: Comité central}

\section{Yvonne Gilli sortante}

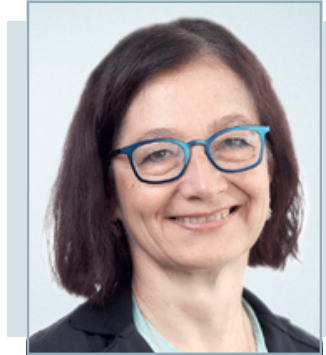

Titre: Dre méd.

Date de naissance: 7.3.1957

Domicile: Wil SG

Lieu d'origine: Neudorf; Beromünster

Etat civil: divorcée, 3 enfants

Courriel: yvonne.gilli@fmh.ch

Titre de spécialiste: médecine interne générale

\section{Affiliation à des sociétés médicales}

- Depuis 2014: Médecins de famille et de l'enfance Suisse (mfe)

- Depuis 2007: medswiss.net - Association suisse des réseaux de médecins

- Depuis 2005: Société suisse de médecine interne générale (SSMIG)

- Depuis 2001: Société suisse de gynécologie et obstétrique (SSGO)

- Depuis 1996: Société médicale du canton de Saint-Gall

- Depuis 1996: Société suisse d'ultrasons en médecine (SSUM)

- Depuis 1996: Association des sociétés médicales suisses d'acupuncture et de médecine chinoise (ASA)

- Depuis 1996: Société suisse des médecins homéopathes (SSMH)

- Depuis 1996: Médecins en faveur de l'environnement (MfE)

- Depuis 1992: FMH

- Depuis 1992: Association suisse des médecins assistant(e)s et chef(fe)s de clinique (ASMAC)

\section{Liens d'intérêts}

- Depuis 2019: membre du Conseil d'administration d'AD Swiss Net SA

- Depuis 2019: membre du Conseil d'administration d'ats-tms SA

- Depuis 2019: membre du Conseil d'administration de Health Info Net SA

- Depuis 2013: présidence du Conseil de fondation de Santé sexuelle suisse

- Depuis 2007: membre du Conseil d'administration de xundart AG

\section{Autres qualifications}

- 2019: psychothérapie IBP

- 1979: infirmière diplômée AKP

- 1976: diplôme de commerce

\section{Langues}

Allemand (langue maternelle), anglais (C1), français (C1), italien (B2)

\section{Activité professionnelle actuelle}

- Depuis 1996: activité de médecin de famille dans un cabinet de groupe à Wil SG

- Depuis 2018: chargée de cours MPH, Université de Zurich

- Depuis 2017: fonction médicale dirigeante dans un cabinet de groupe en psychiatrie à Winterthour

- Depuis 2016: co-direction du CAS en santé sexuelle à la Haute école de santé de Lucerne

\section{Parti politique}

Les Verts

\section{Mandats politiques exercés jusqu'à présent}

- 2007-2015: conseillère nationale et membre des commissions suivantes du Conseil national: Commission de la sécurité sociale et de la santé publique, Com- mission de la science, de l'éducation et de la culture, Commission des finances

- 2004-2007: conseillère d'Etat SG, membre de la Commission des finances

- 2000-2005: membre du parlement municipal de Wil SG, membre de la Commission des constructions et présidence de la Commission des transports

\section{Mandats de politique professionnelle}

- Depuis 2016: membre du Comité central de la FMH, responsable du département Numérisation/eHealth

\section{Expérience de direction}

- Depuis 2007: création, organisation et gestion (direction et membre du Conseil d'administration) du réseau de médecins xundart AG (réseau mixte comprenant des médecins de famille, des médecins agréés et des spécialistes)

- Depuis 2013: présidente du Conseil de fondation de Santé sexuelle suisse (organisation faîtière des services communaux et cantonaux de planning familial)

- 2014-2017: présidence de la "Patientenstelle Ostschweiz»

- 2012-2015: vice-présidence du groupe des Verts au Parlement fédéral

- 2011-2014: cours de leadership pour les femmes dans des fonctions dirigeantes; formation interdisciplinaire organisée sous forme d'intervisions (240 h)

- 2007-2012: présidence du parti cantonal des Verts

\section{Compétences, motivation et objectifs}

"Ce sont les vents contraires qui font s'élever le cerfvolant»: ce proverbe chinois résume très bien ma motivation. La profession médicale est soumise à de gros défis en raison de la forte pression politique. Au Comité central, notre façon de travailler a montré que l'engagement et l'esprit d'équipe sont les clés du succès: la structure tarifaire TARCO a été déposée, le département Numérisation/ eHealth a été réorganisé dans le but de proposer des prestations utiles aux médecins.

Ma longue expérience politique m'a permis de construire un réseau interpartis. J'ai occupé diverses fonctions dirigeantes qui m'ont appris à accompagner les changements structurels et la poursuite de ma formation m'a permis de créer des ponts entre les professions.

Enfin, les vents contraires ne manqueront pas: budget global, surrégulation, pénurie de spécialistes... La FMH doit rester un acteur central de la politique de la santé! Pour moi, il faut continuer à s'engager pour que la profession médicale reste attrayante indépendamment du lieu de travail, de l'organisation du travail, de la spécialité ou du sexe. 


\section{Candidature: Comité central}

\section{Monique Lehky Hagen}

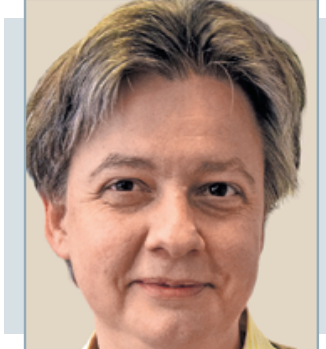

Titre: Dre méd.; Executive MBA focus healthcare

Date de naissance: 29.12.1971

Domicile: Brig-Glis

Lieu d'origine: Naters VS; Basel-Riehen

Etat civil: 3 enfants (1999-2003)

Courriel: lehkyhagen@hin.ch

Titre de spécialiste: médecine interne générale

\section{Affiliation à des sociétés médicales}

- Depuis 2011: Médecins de famille et de l'enfance Suisse (mfe) et Médecins de famille et de l'enfance Suisse (mfe) Valais

- Depuis 2008: Swiss Insurance Medicine (SIM)

- Depuis 2008: Société suisse de médecine manuelle (SAMM)

- Depuis 2007: Société suisse de médecine tropicale et médecine des voyages FMH

- Depuis 2005: Oberwalliser Ärztegesellschaft (OWAeG)

- Depuis 2005: Société suisse d'ultrasons en médecine (SSUM)

- Depuis 2005: Société suisse de médecine interne générale (SSMIG)

- Depuis 2005: Société Médicale du Valais (SMVS)

- Depuis 2002: FMH

- 2002-2005: Association suisse des médecins assistant(e)s et chef(fe)s de clinique (ASMAC)

\section{Liens d'intérêts}

- Depuis 2015: membre du Conseil d'administration de Ctésias SA

- Depuis 2014: membre du Conseil d'administration de NewIndex SA

- Depuis 2011: vice-présidente de la Fondation Humanitaire Sanakvo

\section{Autres qualifications}

- 2016: Executive MBA focus healthcare (UNIL/HEC Lausanne)

- 2010: certificat Appréciation de la capacité de travail (ACT)

- 2008: titre de médecin-expert certifié Swiss Insurance Medicine (SIM)

- 2008: attestation de formation complémentaire Médecine manuelle (SAMM)

- 2007: certificat de médecine tropicale et communautaire (Genève)

- 2005: attestation de formation complémentaire Ultrasonographie abdominale

- 2005: titre de médecine interne générale, Université de Bâle

- 2003: doctorat, Université de Bâle (Institut de Médecine Tropicale)

- 1997: diplôme de médecin, Université de Genève (final court)

- 1994: diplôme de Violon et Virtuosité en Musique de Chambre, Conservatoire de Lausanne

\section{Langues}

Allemand (langue maternelle), français (C2), anglais (C1), italien (B2), tchèque (C2), espagnol (B1)

\section{Activité professionnelle actuelle}

- Depuis 2005: médecine libérale en cabinet privé
- Depuis 2019: médecin-conseil pour l'Organisation régionale du Haut-Valais favorisant la reprise du travail pour chômeurs (OPRA)

- Depuis 2016: chargée de cours de formation sur l'incapacité de travail dans le cadre de la Swiss Insurance Medicine (SIM) pour la Suisse romande

- Depuis 2010: médecin-formateur pour les étudiants de l'Université de Berne

- Depuis 2005: médecin-conseil pour la Société Suisse des Explosifs

- Depuis 2005: médecin-conseil pour les pompiers de Brig-Glis

- Depuis 2005: activité comme médecin-expert (AI jusqu'en 2014, puis expertises juridico-assécurologiques indépendantes)

- Développement de nouveaux concepts interprofessionnels avec suivi scientifique et publication en collaboration avec les pharmaciens valaisans et la Haute école spécialisée de Suisse occidentale (HES-SO) (‘less is more together»)

\section{Parti politique}

aucun

\section{Mandats politiques exercés jusqu'à présent}

- Depuis 2014: membre du Comité Cantonal de la Garde (Kantonale Notfallkommission)

- Depuis 2013: membre du Comité du Service de Coordination socio-sanitaire valaisan (SECOSS)

- 2012-2015: membre de la commission cantonale de la planification hospitalière valaisanne

- 2011: présidente du comité référendaire des médecins valaisans (politiquement indépendant) contre la loi sur les établissements et institutions sanitaires cantonale (LEIS), avec victoire historique à la votation populaire

\section{Mandats de politique professionnelle}

- Depuis 2018: co-présidente de la Conférence des Sociétés Cantonales de Médecine (CCM)

- Depuis 2018: représentante de la Société Médicale de la Suisse Romande (SMSR) au Groupe de travail Laboratoire de la FMH

- Depuis 2013: membre du comité de la Conférence des Sociétés Cantonales de Médecine (CCM)

- Depuis 2013: membre du comité de la Société Médicale de la Suisse Romande (SMSR)

- Depuis 2013: présidente de la Société médicale du Valais (SMVS) 
- Depuis 2011: membre du Comité Médecins de famille et de l'enfance Suisse (mfe) Valais

- Depuis 2010: membre du comité de la Société médicale du Valais (SMVS)

- 2018-2019: représentante de la Conférence des Sociétés Cantonales de Médecine (CCM) au Bureau des tarifs de la FMH

- 2017-2019: membre du groupe tarifaire de la Conférence des Sociétés Cantonales de Médecine (CCM)

- 2017-2018: membre actif de l'alliance SOS-Santé contre la restriction de la durée de consultation par le Conseil fédéral

- 2015-2017: représentante Société Médicale du Valais (SMVS) dans les workshops tarifaires de la FMH pour TARVISION

- 2011-2013: vice-présidente de la Société médicale du Valais (SMVS)

\section{Expérience de direction}

- Depuis 2016: gestion et direction principale du projet tarifaire Société médicale du Valais (SMVS) avec résiliation de la valeur du point TARMED et obtention d'une augmentation dès 2017

- Depuis 2014: participation active à la mise sur pied et au financement d'un service d'urgence commun entre les médecins de premier recours et l'Hôpital du Haut-Valais (HANOW)

- Depuis 2013: présidente Société médicale du Valais (SMVS) - réorganisation et renouvellement du fonctionnement et de la stratégie de communication de la Société médicale du Valais (SMVS) et repositionnement stratégique

- 2018-2019: organisation et coordination de conférences stratégiques bilingues interprofessionnelles Planète Santé Martigny et gestion du projet historique ‘ 175 ans SMVS`

- 2018: Conférence des Sociétés Cantonales de Médecine (CCM) - coordination des sociétés médicales affiliées, organisation d'une journée stratégique commune e-health, protection des données, analyses statistiques 2019, lancement de plusieurs projets stratégiques (coordination échanges de données SASIS, optimalisation des pools de données en rapport avec les analyses d'économicité, évaluation d'une caisse-maladie des médecins)
- 2012-2015: membre du Conseil d'administration de l'Hôpital du Valais: réorganisation interne importante avec remise sur pied du collège des médecinscadres comme instance consultative et participative, influence majeure sur la relocalisation de l'Hôpital du Haut-Valais de Viège à Brigue

- 2012-2015: membre des sous-commissions «Audit internes et `Organisation` du Conseil d’Administration de l'Hôpital du Valais

- 2011: direction, gestion et organisation du comité référendaire et de la campagne réussie contre la loi sur les établissements et institutions sanitaires cantonale (LEIS) avec introduction du collège des médecins dans cette loi cantonale

\section{Compétences, motivation et objectifs}

Par mes engagements et ma participation active à plus de 100 consultations au niveau cantonal et fédéral, j'ai acquis de bonnes connaissances des différentes structures de notre système sanitaire suisse, tant dans le domaine ambulatoire qu'hospitalier. Avec le virage ambulatoire, la digitalisation accrue, l'avidité de surveillance et de collectes de données et les pressions économiques majeures, d'immenses jeux de pouvoir sont en cours. Ils mettent à risque notre liberté de traiter nos patients dans le respect de nos principes et de l'éthique médicale. Il devient d'autant plus crucial de coordonner nos efforts pour maintenir les conditions-cadres nécessaires pour une médecine indépendante, éthique et de qualité au service de nos patients. Si la Chambre médicale le souhaite, je suis prête à m'engager pour un repositionnement rapide de la FMH en tant que partenaire compétent, proactif et respecté dans la politique de santé dans cette optique. 


\section{Candidature: Comité central}

\section{Jürg Lymann}

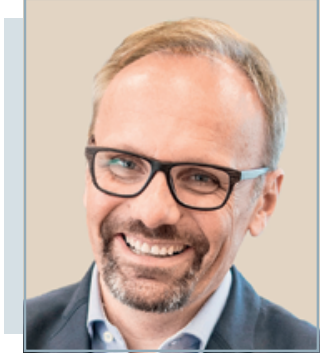

Titre: Dr méd:; MAS FHO

Date de naissance: 22.6.1963

Domicile: Walenstadt

Lieu d'origine: RoggwilTG

Etat civil: marié, 4 enfants

Courriel: jlymann@hin.ch; juerg.lymann@srrws.ch

Titre de spécialiste: gynécologie et obstétrique

\section{Affiliation à des sociétés médicales}

- Depuis 2016: Konferenz der Ostschweizer Ärztegesellschaften (K-OCH)

- Depuis 2016: Verband Deutschschweizer Ärztegesellschaften (VEDAG)

- Depuis 2005: Association des médecins dirigeants d'hôpitaux de Suisse (AMDHS)

- Depuis 2001: Société suisse d'ultrasons en médecine (SSUM), section gynécologie et obstétrique

- Depuis 2000: Verein der Ostschweizer Gynäkologen

- Depuis 1999: Société suisse de gynécologie et d'obstétrique, gynécologie suisse (SSGO)

- Depuis 1999: Société suisse de sénologie (SSS)

- Depuis 1999: Société des médecins du canton de Saint-Gall

- Depuis 1990: FMH

- Depuis 1989: Association suisse des médecins assistant(e)s et chef(fe)s de clinique (ASMAC)

\section{Liens d'intérêts}

aucun

\section{Autres qualifications}

- 2006-2008: diplôme postgrade MAS Health Service Management, Haute école spécialisée de la Suisse orientale (FHO), Saint-Gall

\section{Langues}

Allemand (langue maternelle), italien (C1), français (B2-C1), anglais (B2)

\section{Activité professionnelle actuelle}

- Depuis 1999: médecin-chef à la Frauenklinik Walenstadt, département Frauenkliniken (SRRWS), y c. direction du Brustzentrum Walenstadt en qualité de partenaire de coopération du Brustzentrum St.Gallen-Grabs

- Depuis 1999: propriétaire d'un cabinet de gynécologie à Walenstadt

\section{Parti politique}

aucun

\section{Mandats politiques exercés jusqu’à présent}

- Depuis 2016: membre du conseil de santé du canton de Saint-Gall

\section{Mandats de politique professionnelle}

- Depuis 2018: président Konferenz der Ostschweizer Ärztegesellschaften (K-OCH)

- Depuis 2016: président de la Société des médecins du canton de Saint-Gall

- Depuis 2015: membre du Comité de la Société des médecins du canton de Saint-Gall

- 2010-2013: président Ärzteverein Werdenberg-Sarganserland

- 2007-2016: membre du Comité Ärzteverein Werdenberg-Sarganserland

\section{Expérience de direction}

- Depuis 2010: présidence d'organisations de politique professionnelle (cf. ci-avant)

- Depuis 1999: médecin-chef à la Frauenklinik Spital Walenstadt (SRRWS); nombreuses années comme membre de la direction hospitalière, dont plusieurs comme président suppléant

- Depuis 1999: propriétaire d'un cabinet de gynécologie à Walenstadt

\section{Compétences, motivation et objectifs}

En tant que président d'une société cantonale de médecine et de l'ensemble des sociétés de médecine de Suisse orientale $(\mathrm{K}-\mathrm{OCH})$, j'ai une grande expérience en politique professionnelle. Défendre les intérêts d'un groupe nécessite de bien connaître les processus politiques et de savoir communiquer avec les responsables politiques. Grâce à mon expérience, à la fois en tant que médecin installé et médecin dirigeant en hôpital, j’ai l'habitude du travail en équipe et de la conduite de personnel; de plus, j'ai l'avantage de connaître le quotidien des deux groupes de médecins dans différentes parties du pays.

Ma priorité est d'encourager la cohésion et la coalition du corps médical dans toute sa diversité. Pour cela, il faut observer des règles du jeu claires et prendre en compte les cultures de toutes les parties du pays. J'entends défendre des conditions favorables afin que la profession de médecin reste intéressante et attractive et que les patients continuent de bénéficier d'une bonne prise en charge médicale dans l'ensemble du pays. Cela comprend des postes de formation pré- et postgraduée flexibles en nombre suffisant dans les hôpitaux et les cabinets médicaux de même qu'une tarification équitable. 


\section{Candidature: Comité central}

\section{Michel Matter sortant}

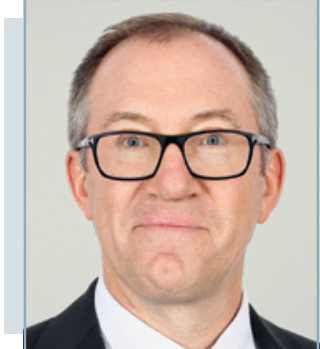

Titre: Dr méd.

Date de naissance: 31.12 .1964

Domicile:Thônex

Lieu d'origine: Genève

Etat civil: marié

Courriel: michel.matter@fmh.ch

Titre de spécialiste: ophtalmologie

\section{Affiliation à des sociétés médicales}

- Depuis 2014: Société médicale de la Suisse romande (SMSR)

- Depuis 2005: Société médicale de Genève

- Depuis 2001: Société suisse d'ophtalmologie

- Depuis 2001: Association des médecins du canton de Genève (AMGe)

- Depuis 1993: FMH

- Membre de diverses sociétés suisses, françaises et américaines d'ophtalmologie

\section{Liens d'intérêts}

- Depuis 2018: membre du Conseil de Fondation PAT-BVG (prévoyance)

- Depuis 2018: membre du Conseil d'administration de FMH Services

- Depuis 2018: membre du Conseil de Fondation de Eyesight4life (Haïti, malvoyance)

- Depuis 2016: membre du Conseil de Direction de la Fédération des entreprises romandes (FER), Genève

- Depuis 2015: membre du Comité de la Fondation PRISM, Genève

- Depuis 2014: membre du Conseil d'administration des Hôpitaux Universitaires de Genève (HUG)

- Depuis 2014: membre de la Commission quadripartite (droits de pratique), Genève

- Depuis 2005: membre du Comité de l'Association du Bien des Aveugles (ABA), Genève

\section{Autres qualifications}

aucune

\section{Langues}

Français (langue maternelle), allemand (B2), anglais (C1)

\section{Activité professionnelle actuelle}

- Depuis 2001: ophtalmologue en pratique privée à Genève

\section{Parti politique}

Vert'libéraux

\section{Mandats politiques exercés jusqu'à présent}

- Depuis 2019: Conseiller national, Commission des Finances

\section{Mandats de politique professionnelle}

- Depuis 2018: vice-président de la FMH

- Depuis 2017: membre du Comité central de la FMH, responsable du département Prestations et développement professionnel

- Depuis 2014: président de l'Association des médecins du canton de Genève (AMGe)

- Depuis 2014: membre du Comité de la Société médicale de la Suisse romande (SMSR)

\section{Expérience de direction}

- Politique professionnelle, voir ci-dessus

\section{Compétences, motivation et objectifs}

Je souhaite poursuivre l'activité exercée au sein du Comité central de la FMH depuis octobre 2017 et pouvoir continuer à défendre l'ensemble des membres de notre Fédération. Les équilibres sont importants et les liens avec les organisations de base ainsi qu'avec les sociétés médicales sont l'une de mes priorités. La qualité de la médecine doit être préservée, son efficience accrue et la formation doit particulièrement être soutenue. Les rapports intergénérationnels, l'égalité et la lutte contre toute forme de sexisme font partie des préoccupations et du travail de mon département. 
Candidature: Comité central

\section{Carlos Beat Quinto sortant}

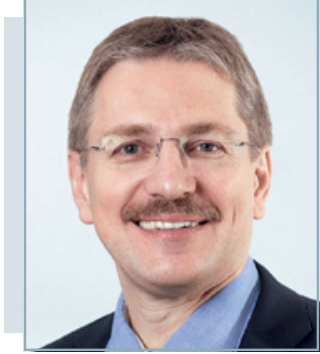

Titre: Dr méd.; MPHH

Date de naissance: 8.9.1966

Domicile: Bâle

Lieu d'origine: Widnau SG

Etat civil: marié, 1 enfant

Courriel: carlos.quinto@fmh.ch; dr.quinto@ hin.ch

Titre de spécialiste: médecine interne générale

\section{Affiliation à des sociétés médicales}

- Depuis 2018: Société suisse des médecins spécialistes en prévention et santé publique (SPHD)

- Depuis 2014: Médecins de famille et de l'enfance Suisse (mfe)

- Depuis 2007: Société suisse d'évaluation (SEVAL)

- Depuis 2007: European Evaluation Society (EES)

- Depuis 2003: Vereinigung der Hausärztinnen und Hausärzte beider Basel (VHBB)

- Depuis 2003: Hausärzteverein Angenstein (HVA)

- Depuis 2002: Swiss Society for Public Health (SSPH)

- Depuis 2002: The European Public Health Association (EUPHA)

- Depuis 1999: Société suisse de médecine interne générale (SSMIG)

- Depuis 1999: Société de médecine de Bâle-Campagne

- Depuis 1992: FMH

- Depuis 1992: Association suisse des médecins assistant(e)s et chef(fe)s de clinique (ASMAC)

\section{Liens d'intérêts}

- Depuis 2019: Conseil d'administration du centre de confiance syndata AG, ancien membre de la direction

- Depuis 2018: Comité Alliance pour la santé en Suisse

- Depuis 2016: Conseil de fondation Promotion Santé Suisse

- Depuis 2016: Conseil scientifique Santé publique Suisse

- 2017-2020: Comité consultatif du programme de promotion «Interprofessionnalité dans le domaine de la santé» de l'OFSP

\section{Autres qualifications}

- Depuis 2007: modérateur de cercles de qualité

- 2007: Master of Public Health

\section{Langues}

Allemand (langue maternelle), anglais (B2), français (B2), espagnol (B2), néerlandais (B2)

\section{Activité professionnelle actuelle}

- Depuis 1999: activité principale indépendante; associé dans le cabinet de groupe Pfeffingen BL, en collaboration avec les Drs J. Fritschi, A. Käppeli et S. Urban

- Depuis 2014: activité annexe: enseignement au Centre universitaire pour la médecine de famille des deux Bâles

- Depuis 2009: activité annexe: «Senior scientific collaborator», Institut tropical et de santé publique Suisse (Swiss TPH), département Epidemiology and Public Health, Chronic Disease Epidemiology Unit

\section{Parti politique}

aucun

\section{Mandats politiques exercés jusqu'à présent} aucun

\section{Mandats de politique professionnelle}

- Depuis 2016: membre du Comité central de la FMH, responsable du département Santé publique, professions de la santé et produits thérapeutiques

- Depuis 2012: membre du Comité de la Société des médecins du canton de Bâle-Campagne

- Depuis 2012: membre de la rédaction de la revue Synapse (www.emh.ch)

- 1992-2003: membre du Comité, caissier et ensuite viceprésident de l'Association suisse des médecins assistan$\mathrm{t}(\mathrm{e}) \mathrm{s}$ et chef(fe)s de clinique (ASMAC), section bâloise

\section{Expérience de direction}

- Depuis 2016: membre du Comité central de la FMH, responsable du département Santé publique, professions de la santé et produits thérapeutiques

- Depuis 2006: gestion autonome de projets d'évaluation de portée nationale

- Depuis 1999: gestion du personnel au sein du cabinet de groupe

- 2004-2016: activité en tant que médecin d'état-major (capitaine): organisation du service sanitaire

\section{Compétences, motivation et objectifs}

Depuis 2016, je suis responsable du département Santé publique, professions de la santé et produits thérapeutiques au sein du Comité central et à la tête d'une équipe motivée. Nous avons réussi à intégrer à nouveau des représentants des trois régions linguistiques à la Commission de santé publique de la FMH et à renforcer la position de la FMH au niveau national dans ce domaine. En ce qui concerne les assistantes médicales, la nouvelle ordonnance révisée sur la formation professionnelle initiale a été mise en vigueur et dans le domaine des produits thérapeutiques, la sécurité des patients et la sécurité de l'approvisionnement restent des sujets cruciaux.

$\mathrm{Au}$ sein de la FMH, je me considère comme un tisseur de liens entre la médecine générale et la santé publique, entre le domaine médical et le domaine public tant au niveau individuel que sociétal. La question de la relève médicale me tient à cœur et c'est pourquoi je représente la FMH à la CIMS et au Swiss Medical Weekly. La relation médecin-patient est essentielle à mes yeux et il s'agit de contribuer à créer des conditions-cadres qui permettent aux médecins de continuer à avoir du plaisir dans l'exercice de leur profession. Voici les sujets pour lesquels je souhaite continuer à m’engager au sein du Comité central. 


\section{Candidature: Comité central}

\section{Urs Stoffel sortant}

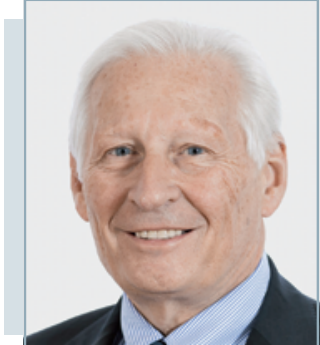

Titre: Dr méd.

Date de naissance: 5.7 .1952

Domicile: Kilchberg ZH

Lieu d'origine: Arbon

Etat civil: marié, 2 enfants

Courriel: urs.stoffel@hin.ch; urs.stoffel@fmh.ch

Titre de spécialiste: chirurgie

Affiliation à des sociétés médicales

- Depuis 2004: Société suisse de chirurgie (SSC)

- Depuis 2004: Association suisse des médecins avec activité chirurgicale et invasive (FMCH)

- Depuis 1998: Société allemande de coloproctologie

- Depuis 1996: Société suisse de chirurgie générale et de traumatologie (SSCGT)

- Depuis 1995: Société des médecins du canton de Zurich

- Depuis 1989: Association Suisse pour la Chirurgie Laparoscopique et Thoracoscopique (ASCLT)

- Depuis 1985: FMH

- 2002-2016: Société des médecins du canton d’Argovie

\section{Liens d'intérêts}

- Depuis 2015: Conseil d'administration de la RehaClinic Zurich SA

- Depuis 2012: président du Conseil d'administration de NewIndex SA (sur mandat du Comité central de la FMH)

- Depuis 2012: président du Conseil d'administration de HIN SA (sur mandat du Comité central de la FMH)

- Depuis 2011: Conseil de fondation de la "Fondation Gaydoul»

- Depuis 2010: Conseil de fondation de l'ancienne clinique d'altitude Sanitas à Davos

\section{Autres qualifications}

- 1971: diplôme de commerce

\section{Langues}

Allemand (langue maternelle), français (B1), anglais (B2), italien (B1)

\section{Activité professionnelle actuelle}

- 1996: spécialiste en chirurgie générale dans le cabinet de groupe Enge à Zurich et médecin agréé dans quatre cliniques (Klinik Hirslanden, Klinik im Park, Privatklinik Bethanien et Seespital Kilchberg)

\section{Parti politique}

PLR

\section{Mandats politiques exercés jusqu'à présent} aucun

\section{Mandats de politique professionnelle}

- Depuis 2012: membre du Comité central

- Depuis 2015: responsable du département Médecine et tarifs ambulatoires

- 2012-2016: responsable du département eHealth, infrastructure de sécurité et collecte de données

- 2006-2011: co-président de la Conférence des sociétés cantonales de médecine (CCM)

- 2002-2014: président de la Société des médecins du canton de Zurich (AGZ)

- 2004: délégué de la FMH au sein du bureau de neutralité des coûts TARMED

\section{Expérience de direction}

- Voir sous «Liens d'intérêts»

- Voir sous «Activité professionnelle actuelle»

- Voir sous «Mandats de politique professionnelle»

\section{Compétences, motivation et objectifs}

Au cours des quatre dernières années, nous avons, avec l'équipe du Service tarifaire, travaillé d'arrache-pied sur le tarif ambulatoire. Le vaisseau TARDOC a atteint son premier objectif et a été remis au Conseil fédéral. Une grande partie de notre activité a également porté sur l'intervention tarifaire, le monitorage, le relevé MAS et le tarif de laboratoire. Nous avons organisé des rencontres régulières entre les présidents des sociétés cantonales et le cockpit, créant ainsi deux importantes plateformes qui ont permis de réunir à nouveau le corps médical autour des questions tarifaires.

Mais nous sommes encore loin du but et certains obstacles demeurent. Comme je ne veux pas remettre à ma ou mon successeur-e un projet qui n'a pas encore abouti, je suis prêt à remettre l'ouvrage sur le métier pour la prochaine législature. Si la Chambre médicale m'accorde sa confiance, confirme l'exception relative à l'âge limite, il me tient à cœur, afin d'assurer la continuité, de former une ou un successeur-e pour la reprise de ce département qui demande un grand savoir-faire et une grande disponibilité. 


\section{Candidature: Comité central}

\section{Jürg Unger sortant}

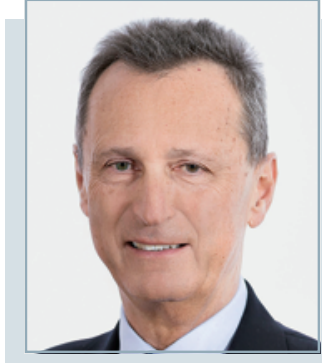

Titre: Dr méd.

Date de naissance: 30.4 .1955

Domicile: Zurich

Lieu d'origine: Zurich; Baden

Etat civil: marié, 4 enfants

Courriel: juerg.unger@fmh.ch

Titre de spécialiste: psychiatrie et psychothérapie d'enfants et d'adolescents

\section{Affiliation à des sociétés médicales}

- Depuis 2013: Association suisse des médecins-chefs en psychiatrie des enfants et adolescents (AMCPEA)

- Depuis 2008: Société des médecins du canton de Zurich (AGZ)

- Depuis 2005: Association des médecins dirigeants d'hôpitaux de Suisse (AMDHS)

- Depuis 2002: Société suisse de psychiatrie (SSP)

- Depuis 1988: Société suisse de psychiatrie et psychothérapie de l'enfant et de l'adolescent (SSPPEA) et dès sa fondation Foederatio Medicorum Psychiatricorum et Psychotherapeuticorum (FMPP)

- Depuis 1981: FMH

\section{Liens d'intérêts}

- Depuis 2016: membre du Comité de Swiss Mental Health Care (SMHC) (jusqu'en juin 2020)

\section{Autres qualifications}

- Diverses formations continues en management

\section{Langues}

Allemand (langue maternelle), français (B1), anglais (C1)

\section{Activité professionnelle actuelle}

- Depuis 2016: activité de consultant dans le domaine de la santé (conseils cliniques, questions organisationnelles et sujets en lien avec la formation, placement de personnel)

\section{Parti politique}

aucun

\section{Mandats politiques exercés jusqu'à présent}

aucun

\section{Mandats de politique professionnelle}

- Depuis 2016: membre du Comité central de la FMH, responsable du département Médecine et tarifs hospitaliers

- Depuis 2013: vice-président de l'Association suisse des médecins-chefs en psychiatrie des enfants et adolescents (AMCPEA)

- 2013-2016: représentant de la FMH dans le groupe de suivi TARPSY de SwissDRG SA

- Jusqu'en 2016: expert aux examens pour le titre de spécialiste en psychiatrie et psychothérapie d'enfants et d'adolescents
- 2009: représentant de la Foederatio Medicorum Psychiatricorum et Psychotherapeuticorum (FMPP) à l’Assemblée des délégués et à la Chambre médicale de la FMH

- 2008-2010: représentant de la Foederatio Medicorum Psychiatricorum et Psychotherapeuticorum (FMPP) dans le groupe de travail eHealth de la FMH

- Quatre ans comme membre du Comité de la Société suisse de psychiatrie et psychothérapie de l'enfant et de l'adolescent (SSPPEA)

- Membre du Comité de la foederatio Paedo medicorum helveticorum (fPmh) (membre fondateur)

\section{Expérience de direction}

- 1996-2004/2009-2016: médecin-chef en psychiatrie d'enfants et d'adolescents dans le canton d'Argovie (100-180 collaborateurs)

- 2004-2006: CEO Psychiatrie Aargau (1000 collaborateurs)

- 1988-1996: chef de clinique à l'Hôpital pédiatrique de Zurich (psychiatrie et protection de l'enfance en chirurgie pédiatrique, pédiatrie, réadaptation)

- 2007-2010: membre du Conseil d'administration du réseau de médecins zmed

- 2003-2009: membre de différents conseils de fondation

\section{Compétences, motivation et objectifs}

Depuis quatre ans, j'ai l'immense plaisir de gérer le département Médecine et tarifs hospitaliers de la FMH. Je serais heureux de pouvoir poursuivre cette activité et de continuer à représenter avec succès les intérêts de la FMH au Conseil d'administration de SwissDRG SA. Je serais également ravi de poursuivre l'excellente collaboration entre les deux départements tarifaires au plan stratégique.

Le projet "Coach my Career» dédié à la nouvelle génération de médecins et conduit par plusieurs associations au sein de la FMH me tient particulièrement à cœur. Le rôle du Comité central consiste aussi à favoriser la cohésion interne à tous les niveaux et à renforcer l'influence du corps médical. La question se pose désormais de savoir dans quelle mesure nous, médecins, interviendrons activement dans l'avenir des hôpitaux. L'enquête annuelle auprès du corps médical assure une bonne présence médiatique à la FMH et permet d'obtenir de précieuses informations pour le positionnement de notre association. 


\section{Candidature: Comité central}

\section{Alexander Zimmer}

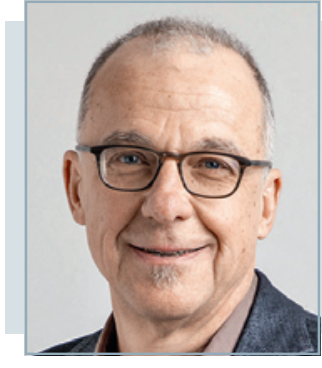

Titre: Dr méd.

Date de naissance: 9.7.1961

Domicile: Soleure

Lieu d'origine: Zurich

Etat civil: En couple avec la co-présidente de la Fédération

suisse des psychologues (FSP), 2 enfants

Courriel: azimmer@hin.ch

Titre de spécialiste: psychiatrie et psychothérapie

\section{Affiliation à des sociétés médicales}

- 1999-2005/depuis 2014: Société des médecins du canton de Soleure (GAeSo)

- Depuis 1998: Société suisse de psychiatrie et psychothérapie (SSPP) et Foederatio Medicorum Psychiatricorum (FMPP)

- 1997-2005/depuis 2014: Société de psychiatrie et psychothérapie du canton de Soleure (GPPSo)

- Depuis 2010: Société suisse de psychiatrie de consultation-liaison et psychosomatique (SSCLP)

- Depuis 2010: Swiss Society of Addiction Medicine (SSAM)

- Depuis 1998: FMH

- 2006-2013: Société médicale de Bâle-Campagne (AeGBL) et groupe spécialisé psychiatrie Bâle-Campagne

- 2002-2013: Association des médecins dirigeants d'hôpitaux de Suisse (AMDHS)

- 1991-2000: Association suisse des médecins assistant(e)s et chef(fe)s de clinique (ASMAC)

\section{Liens d'intérêts}

- Depuis 2019: vice-président du Spitalrat Integrierte Psychiatrie Winterthur Zürcher Unterland (ipw)

- Depuis 2017: membre de l'Académie suisse de médecine psychosomatique et psychosociale (ASMPP)

\section{Autres qualifications}

- 2008-2009: nouveaux concepts de gestion de conseils d'administration, Institut für Führung und Personalmanagement, Prof. Martin Hilb, Université de Saint-Gall

- 1999-2000: formation continue en management au college M, Berne

\section{Langues}

Allemand (langue maternelle), français (B2), anglais (B2)

\section{Activité professionnelle actuelle}

- Depuis 2014: cabinet privé à Soleure

\section{Parti politique}

aucun

\section{Mandats politiques exercés jusqu'à présent}

aucun

\section{Mandats de politique professionnelle}

- Depuis 2016: membre de la Chambre médicale de la FMH

- Depuis 2016: président de la Commission permanente des tarifs (CPT) de la Foederatio Medicorum Psychiatricorum et Psychotherapeuticorum (FMPP)

- Depuis 2016: membre du Bureau des tarifs de la FMH et de l'organe de pilotage cockpit TARCO

- Depuis 2016: membre du Comité de la SSPP
- Depuis 2016: membre du Comité de la Foederatio Medicorum Psychiatricorum et Psychotherapeuticorum (FMPP)

- Depuis 2014: membre du groupe de travail eHealth de la FMH

- Depuis 2014: membre suppléant du groupe de travail Données, démographie et qualité (DDQ) de la FMH

- Depuis 2012: membre de la Commission de gestion (CdG) de la FMH

- 2014-2015: membre de la taskforce de la FMH sur la stabilisation budgétaire

- 2009-2014: membre du Comité de l'Association suisse des médecins-cheffes et médecins-chefs en psychiatrie (ASMP)

- 2006-2014: membre du Comité du groupe spécialisé Psychiatrie et psychothérapie Bâle-Campagne

- 2006-2014: membre de l'Assemblée des délégués de la Société suisse de psychiatrie et psychothérapie (SSPP) et de la Foederatio Medicorum Psychiatricorum (FMPP)

- 2005-2015: président de la Commission permanente des tarifs de la Foederatio Medicorum Psychiatricorum et Psychotherapeuticorum (FMPP)

\section{Expérience de direction}

- Depuis 2019: vice-président du Spitalrat Integrierte Psychiatrie Winterthur - Zürcher Unterland (ipw)

- Depuis 2014: cabinet de groupe Kreuzackerpark Soleure

- 2006-2014: médecin-chef et membre de la direction de Psychiatrie Bâle-Campagne

- 2000-2005: médecin dirigeant du Service de psychiatrie du canton de Soleure

- 1997-1999: chef de clinique au Psychiatrisches Ambulatorium und Poliklinik für Heroingestützte Behandlung (HeGeBe) à Soleure

\section{Compétences, motivation et objectifs}

Mes huit années d'engagement au sein de la Commission de gestion et des différents groupes de travail de la FMH mont permis d'acquérir de solides connaissances dans les multiples sujets traités par notre organisation professionnelle et d'établir des liens avec un grand nombre de personnes impliquées dans ces sujets. Je souhaiterais désormais mettre ces compétences au service du Comité central, l'organe stratégique de la FMH. La collaboration avec les sociétés cantonales et les sociétés de discipline médicale, les organisations de base et nos partenaires du domaine de la santé reste passionnante. En tant que médecin, j'aimerais que le point de vue et les prises de position du corps médical soient mieux entendus par le public et les politiques, même s'il faut aussi savoir se montrer conciliant pour aboutir à des solutions constructives. De plus, il est essentiel d'assurer de bonnes conditions-cadres aux médecins hospitaliers et installés pour qu'ils puissent continuer à contribuer à la qualité élevée de notre système de santé. 


\section{Candidature: Commission de gestion}

\section{Nicola Edoardo Ghisletta}

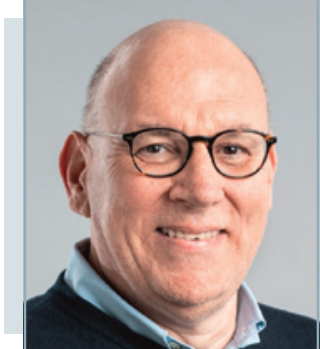

Titre: Dr méd.

Date de naissance: 1.2.1964

Domicile: Cureglia

Lieu d'origine: Camorino

Etat civil: divorcé

Courriel: nighi@bluewin.ch

Titre de spécialiste: chirurgie

\section{Affiliation à des sociétés médicales}

- Depuis 2001: Ordine dei Medici del Canton Ticino (OMCT)

- Depuis 2001: Circolo Medico di Lugano

- Depuis 2001: Société suisse de chirurgie (SSC)

- Depuis 1991: Association suisse des médecins assistant(e)s et chef(fe)s de clinique (ASMAC)

- Depuis 1991: FMH

\section{Liens d'intérêts}

aucun

\section{Autres qualifications}

aucune

\section{Langues}

Italien (langue maternelle), français (C1), allemand (C1), anglais (B2)

\section{Activité professionnelle actuelle}

- Depuis 2019: consultation chirurgicale dans un cabinet de groupe à Manno

- Depuis 2016: consultation chirurgicale en pratique privée à Giubiasco

- Depuis 2007: spécialiste en chirurgie générale avec cabinet privé à la Clinica Luganese Moncucco de Lugano

\section{Parti politique}

PLR

\section{Mandats politiques exercés jusqu'à présent}

- Depuis 2016: membre du Conseil communal et de la Commission de gestion de Cureglia

\section{Mandats de politique professionnelle}

- Depuis 2003: membre du Comité de l'Ordine dei Medici del Canton Ticino (OMCT)

- 2012-2019: président du Gruppo di Chirurgia regionale Ticino

- 2001-2006: membre du Comité de l'Association suisse des médecins assistant(e)s et chef(fe)s de clinique (ASMAC), section Tessin

\section{Expérience de direction}

- 2001-2007: chef de clinique, Hôpital régional de Lugano

- 2007-2019: médecin-adjoint à la Clinica Luganese Moncucco (responsable de la formation des médecins-assistants et de la formation en chirurgie)

- 2008-2014: responsable de l'équipe des médecins du Hockey Club Lugano

- Armée: premier-lieutenant

- Responsable de l'organisation de manifestations de bienfaisance (Golf Club Gerre Losone - B52)

\section{Compétences, motivation et objectifs}

Au cours de mes dix années au Comité de l'Ordine dei Medici del Canton Ticino (OMCT), je me suis souvent frotté à la dimension politique de cette activité au service du corps médical. L'importance des associations médicales m'y est apparue encore plus clairement et c'est pourquoi je suis très motivé à poursuivre mon engagement et à jouer un rôle actif au sein de la Commission de gestion.

Le président de l'OMCT a proposé ma candidature à la succession de la personne qui représente actuellement le Tessin à la Commission de gestion. Je suis convaincu de l'importance de réélire un Tessinois à ce poste.

Doté d'un esprit pragmatique et analytique, de compétences sociales et professionnelles, je souhaite mettre ces atouts au service de l'évaluation des investissements et des dépenses proposés.

Pour toutes ces raisons, je vous invite à soutenir ma candidature à la Commission de gestion. 


\section{Candidature: Commission de gestion}

\section{Jürg Lareida sortant}

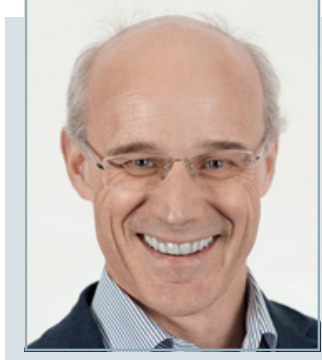

Titre: Dr méd.

Date de naissance: 19.5.1958

Domicile: Aarau

Lieu d'origine: Aarau; Präz

Etat civil: marié, 2 enfants

Courriel: juerg.lareida@ hin.ch

Titre de spécialiste: endocrinologie et diabétologie;

médecine interne générale

\section{Affiliation à des sociétés médicales}

- Depuis 2003: European Group of Graves' Orbitopathy

- Depuis 1998: Endocrine Society

- Depuis 1996: European Society of Endocrinology

- Depuis 1996: Association suisse contre l'ostéoporose

- Depuis 1994: Société des médecins du canton d'Argovie

- Depuis 1992: Société suisse d'endocrinologie et de diabétologie (SSED)

- Depuis 1991: Société suisse de médecine interne générale (SSMIG)

- Depuis 1986: FMH

\section{Liens d'intérêts}

aucun

\section{Autres qualifications}

aucune

\section{Langues}

Allemand (langue maternelle), français (C1), anglais (C1), italien (C1)
Activité professionnelle actuelle

- Depuis 1995: activité en pratique privée

- Depuis 1995: médecin agréé

\section{Parti politique}

aucun

\section{Mandats politiques exercés jusqu'à présent}

- 1991-1997: conseiller communal de la ville d'Aarau, membre de la Commission des finances et de gestion de la ville d'Aarau

- 1999-2004: Commission fiscale de la ville d'Aarau

\section{Mandats de politique professionnelle}

- Depuis 2016: président de la Société des médecins du canton d'Argovie

- Depuis 2013: membre de la Commission de gestion de la FMH

- Depuis 2008: membre de la direction de la Société des médecins du canton d'Argovie

\section{Expérience de direction}

- Politique professionnelle, voir ci-dessus

- Officier de l'Armée suisse (premier-lieutenant)

\section{Compétences, motivation et objectifs}

Cela fait sept ans que je suis membre de la Commission de gestion et je me mets volontiers à disposition pour la prochaine législature. 


\section{Candidature: Commission de gestion}

\section{Philippe Vuillemin sortant}

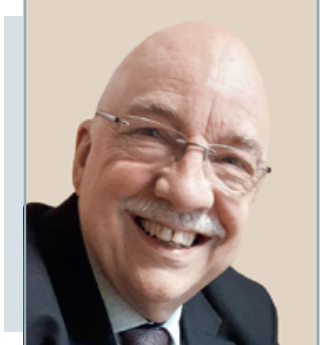

Titre: aucun

Date de naissance: 11.2.1953

Domicile: Lausanne

Lieu d'origine: Lausanne

Etat civil: marié

Courriel: philippe.vuillemin@mac.com

Titre de spécialiste: médecin praticien

\section{Affiliation à des sociétés médicales}

- Depuis 2010: Société médicale de la Suisse romande (SMSR)

- Depuis 1985: Société vaudoise de médecine (SVM)

- Depuis 1980: FMH

\section{Liens d'intérêts}

- Depuis 2009: membre du Comité central Alzheimer suisse

- Depuis 2008: membre du Conseil d'éthique de l'Association vaudoise des établissements médico-sociaux (HeViVa)

\section{Autres qualifications}

aucune

\section{Langues}

Français (langue maternelle), allemand (B2), suisse-allemand (C1), anglais (C1)

\section{Activité professionnelle actuelle}

- Depuis 1985: cabinet médical à Lausanne

- Depuis 2008: membre du Conseil de fondation «Soins Lausanne», Soins à domicile à Lausanne

- Depuis 2002: médecin responsable du groupe d'EMS «ALTAGE» à Yverdonles-Bains

- Depuis 1987: membre du Conseil de fondation et médecin responsable de l'EMS la Rozavère à Lausanne

\section{Parti politique}

PLR

\section{Mandats politiques exercés jusqu'à présent}

- Depuis 2016: vice-président de la Commission de Santé publique du Grand Conseil vaudois
- 2010-2016: membre de la Commission de Santé publique du Grand Conseil vaudois

- 1990-2007/depuis 2010: député au Grand Conseil vaudois

- Depuis 2010: co-président de la commission interparlementaire Vaud-Valais de l'Hôpital Riviera Chablais

- 1998-2007: membre, puis vice-président de la Commission de gestion du Grand Conseil

- 2003-2007: membre de la Commission de la gestion politique de la Banque cantonale vaudoise

\section{Mandats de politique professionnelle}

- Depuis 2018: vice-président de la Commission de gestion de la FMH

- Depuis 2008: délégué de la Société vaudoise de médecine (SVM) à la Chambre médicale de la FMH

- 2011-2016: président de l'Assemblée des délégués de la Société vaudoise de médecine (SVM)

\section{Expérience de direction}

- Différents mandats, comme président de parti, du Conseil communal de Lausanne, de commissions parlementaires, fondations et associations diverses, Conseil des Hospices-CHUV

- Expérience militaire (colonel des troupes sanitaires)

\section{Compétences, motivation et objectifs}

Elu en mai 2018, j'ai repourvu le siège romand. J'assume la vice-présidence de la Commission de gestion de la FMH. Je trouve pleine satisfaction à exercer un mandat exigeant en disponibilités, responsabilités vis-à-vis de notre communauté médicale, et passionnant par les contacts qu'il permet de nouer avec les instances de la FMH. Je désire participer à la continuité de la mission de la Commission de gestion de la FMH, au moment où trois membres la quittent.

Je suis candidat à un nouveau mandat comme membre de la Commission de gestion de la FMH. 


\section{Candidature: présidence de la Commission de déontologie}

\section{Francesca Mainieri sortante}

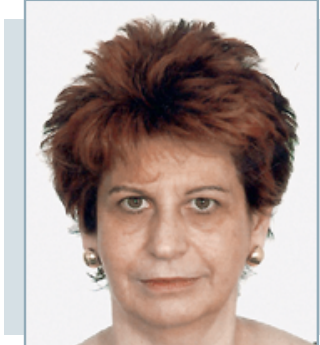

Titre: Dre méd.

Date de naissance: 22.11.1957

Domicile: Muzzano

Lieu d'origine: Zurich

Etat civil: célibataire

Courriel: francesca.mainieri@hin.ch

Titre de spécialiste: médecine interne générale

\section{Affiliation à des sociétés médicales}

- Depuis 2002: Société suisse de médecine interne générale, section du Tessin (SSMIG-TI)

- Depuis 1998: Société suisse d'ultrasons en médecine (SSUM)

- Depuis 1998: Società di Ultrasonologia medica della Svizzera Italiana (SGUMSI)

- Depuis 1997: Société suisse de médecine interne générale (SSMIG)

- Depuis 1991: Ordine dei Medici del Cantone Ticino

- Depuis 1990: Association suisse des médecins assistant(e)s et chef(fe)s de clinique (ASMAC)

- Depuis 1990: FMH

\section{Liens d'intérêts}

aucun

\section{Autres qualifications}

aucune

\section{Langues}

Italien (langue maternelle), allemand (C2), français (B2), anglais (B1)

\section{Activité professionnelle actuelle}

- Depuis 1998: cabinet de médecine interne générale à Paradiso

\section{Parti politique}

aucun

\section{Mandats politiques exercés jusqu'à présent} aucun

\section{Mandats de politique professionnelle}

- Depuis 2006: présidence de la Commission de déontologie de la FMH

- Depuis 2002: présidence de la Société suisse de médecine interne générale, section du Tessin (SSMIG-TI)

\section{Expérience de direction}

- Politique professionnelle, voir ci-dessus 


\section{Candidature: vice-présidence de la Commission de déontologie}

\section{Charles A. Favrod-Coune sortant}

Titre: Dr méd.

Date de naissance: 30.12 .1952

Domicile: Château-d'Cxx

Lieu d'origine: Château-d'ÆEx

Etat civil: marié

Courriel: charles.a.favrod-coune@svmed.ch

Titre de spécialiste: médecine interne générale;

endocrinologie/diabétologie

\section{Affiliation à des sociétés médicales}

- Depuis 1997: Société suisse d'endocrinologie et diabétologie (SSED)

- Depuis 1990: Société Vaudoise de Médecine (SVM)

- Depuis 1986: Société Française d’Endocrinologie

- Depuis 1978: FMH

\section{Liens d'intérêts}

aucun

\section{Autres qualifications}

- 1990: diplôme en Economie et Administration de la Santé, Université de Lausanne

\section{Langues}

Français (langue maternelle), allemand (C1), anglais (B2), italien (B1)

\section{Activité professionnelle actuelle}

- Depuis 2018: consultation d'endocrinologie-diabétologie au Pôle-Santé du Pays-d'Enhaut à Château-d'Ex

\section{Parti politique}

PLR

\section{Mandats politiques exercés jusqu'à présent}

- 2011-2016: Conseil communal puis Municipalité de Château-d'Ex

\section{Mandats de politique professionnelle}

- Depuis 2015: membre de l'Assemblée des délégués de la Société Vaudoise de Médecine (SVM)

- Depuis 2013: vérificateur des comptes de la Société médicale de la Suisse romande (SMSR)

- Depuis 2012: vice-président de la Commission de déontologie de la FMH
- Depuis 2008: membre de la Chambre médicale de la FMH

- 2008-2012: président de la Société médicale de la Suisse romande (SMSR)

- 2006-2012: membre de l’Assemblée des délégués de la FMH

- 2004-2006: membre de la Commission des finances de la FMH

- 2000-2008: président de la Société Vaudoise de Médecine (SVM)

- Représentation de la FMH à l'Union Européenne des Médecins Spécialistes (Endocrinologie-Diabétologie)

\section{Expérience de direction}

- Présidence ou membre du Conseil d'administration de plusieurs sociétés commerciales et fondations

- Politique professionnelle, voir ci-dessus

\section{Compétences, motivation et objectifs}

Une intense participation aux associations professionelles médicales m'a donné une compréhension étendue de la médecine et de la vie associative médicale. Je suis donc à même de saisir les problèmes de l'activité médicale dans toute leur complexité. Ayant pratiqué de nombreuses années en hôpital puis en propre cabinet, je suis à même d'avoir un éclairage pratique sur les problèmes de déontologie.

Dans un monde qui change, je suis attentif à l'émergence des nouvelles problématiques qui constituent de nouveaux challenges éthiques. Ce sont par exemple le développement de l'information sur internet, les exigences accrues du consentement éclairé, la médecine prédictive, le poids toujours plus grand des contraintes juridiques.

Après deux périodes de quatre ans, mon expérience de la Commission de déontologie pourra rester au service des médecins de la FMH. 


\section{Candidature: vice-présidence de la Commission de déontologie}

\section{Beat Niklaus Manser sortant}

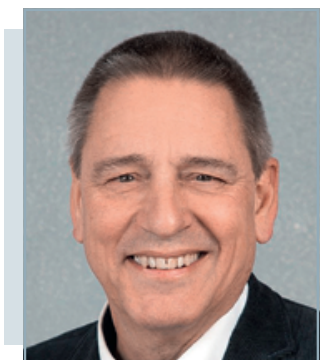

Titre: Dr méd.

Date de naissance: 11.4 .1956

Domicile: Eich LU

Lieu d'origine: Appenzell

Etat civil: marié

Courriel: manser@tic.ch

Titre de spécialiste: médecine interne générale

\section{Affiliation à des sociétés médicales}

- Depuis 1994: Médecins de famille et de l'enfance Suisse (mfe)

- Depuis 1989: Société suisse de médecine interne générale (SSMIG)

- Depuis 1989: Société médicale du canton de Lucerne

- Depuis 1989: Association suisse des médecins assistant(e)s et chef(fe)s de clinique (ASMAC)

- Depuis 1983: FMH

\section{Liens d'intérêts}

- Depuis 2011: président du Conseil d'administration et membre de la direction de la Notfallpraxis der Hausärzte Sursee AG

- Depuis 2010: membre du Comité Ärzte mit Patientenapotheke APA

- Depuis 2003: président du Conseil d'administration du centre de confiance de Suisse centrale medkey AG

\section{Autres qualifications}

aucune

\section{Langues}

Allemand (langue maternelle), français (B2), anglais (B2), italien (B2)

\section{Activité professionnelle actuelle}

- Depuis 1989: cabinet privé à Eich LU

\section{Parti politique}

aucun

\section{Mandats politiques exercés jusqu'à présent} aucun

\section{Mandats de politique professionnelle}

- Depuis 2016: vice-président de la Commission de déontologie de la FMH

- Depuis 2010: membre du Comité «Ärzte mit Patientenapotheke APA"

- Depuis 1998: mise sur pied du centre de collecte de données de Suisse centrale

- 2004-2008: membre de la direction de la Conférence des sociétés cantonales de médecine (CCM)

- 2004-2008: président de la Société lucernoise de médecine

- 2002: membre du Comité de la Société lucernoise de médecine, responsable tarifaire

- 1997-2001: président de la société de médecine générale du canton de Lucerne

- 1995-2002: membre du Comité de la société de médecine générale du canton de Lucerne

\section{Expérience de direction}

- Armée: ancien lieutenant des troupes sanitaires

- Politique professionnelle: voir ci-dessus

\section{Compétences, motivation et objectifs}

Je souhaite défendre la profession médicale contre les attaques internes et externes. 


\section{Candidature: vice-présidence de la Commission de déontologie}

\section{Jean-Marc Reymond sortant}

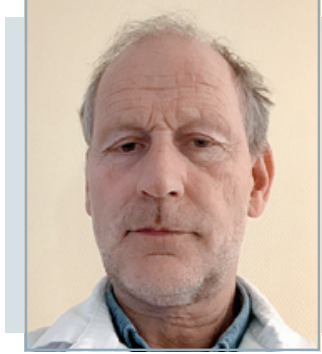

Titre: Dr méd.

Date de naissance: 19.8.1958

Domicile: Genève

Lieu d'origine: Gimel VD

Etat civil: divorcé

Courriel: jmreymond@hin.ch

Titre de spécialiste: médecine interne générale

\section{Affiliation à des sociétés médicales}

- Depuis 2003: Société suisse de médecine interne générale (SSMIG)

- Depuis 1997: Association des médecins du canton de Genève (AMGe)

- Depuis 1984: FMH

\section{Liens d'intérêts}

aucun

\section{Autres qualifications}

aucune

\section{Langues}

Français (langue maternelle), anglais (B2)

\section{Activité professionnelle actuelle}

- Depuis 1997: cabinet privé à Genève

\section{Parti politique}

aucun

\section{Mandats politiques exercés jusqu'à présent} aucun

\section{Mandats de politique professionnelle}

- Depuis 2016: vice-président de la Commission de déontologie de la FMH

- Depuis 2016: président de la Commission de déontologie et conciliation de l'Association des médecins du canton de Genève (AMGe)

\section{Expérience de direction}

- Politique professionnelle, voir ci-dessus

\section{Compétences, motivation et objectifs}

Intérêt pour les questions déontologiques de la profession médicale dans un contexte de changement de plus en plus rapide de l'exercice privé de la médecine. Douze ans au sein de la Commission de déontologie de l'Association des médecins du canton de Genève et deux ans au sein de la Commission de déontologie de la FMH afin de préserver une vision éthique de la profession de médecin tant au niveau des patients que des confrères. 


\section{Candidature: vice-présidence de la Commission de déontologie}

\section{Mathias Wenger sortant}

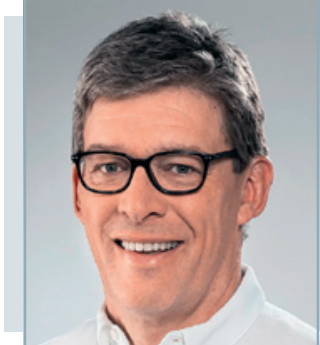

Titre: Dr méd.

Date de naissance: 29.3.1956

Domicile: Frauenfeld

Lieu d'origine: Blumenstein BE

Etat civil: divorcé

Courriel: mathias.wenger@schlossberg-aerzte.ch

Titre de spécialiste: médecine interne générale

\section{Affiliation à des sociétés médicales}

- Depuis 2004: Société suisse de médecine du travail (SSMT)

- Depuis 1991: Société suisse de médecine interne générale (SSMIG)

- Depuis 1990: Société des médecins du canton de Thurgovie

- Depuis 1988: Association suisse des médecins assistant(e)s et chef(fe)s de clinique (ASMAC)

- Depuis 1983: FMH

\section{Liens d'intérêts}

- Depuis 2014: membre du Conseil de fondation et du Comité de placement de pro Medico

\section{Autres qualifications}

- 2011: DAS en gestion de santé, Université de Berne

\section{Langues}

Allemand (langue maternelle), anglais (C1), français (B2)

\section{Activité professionnelle actuelle}

- Depuis 2014: médecin de famille à Islikon, responsable du site d'Islikon au sein du Schlossberg Ärztezentrum AG à Frauenfeld

\author{
Parti politique \\ PDC
}

\section{Mandats politiques exercés jusqu'à présent} aucun

\section{Mandats de politique professionnelle}

- Depuis 2014: vice-président de la Commission de déontologie de la FMH

- 2007-2011: caissier de la Société suisse de médecine du travail (SSMT)

- 2003-2005: co-président de la Verband Deutschschweizer Ärztegesellschaften (VEDAG)

- 2000-2005: président de la Société des médecins du canton de Thurgovie

\section{Expérience de direction}

- 2011-2014: médecin cantonal de Thurgovie

- 2007-2011: directeur Schlossberg Ärztezentrum (env. 60 collaborateurs)

- 2005-2010: médecin cantonal suppléant de Thurgovie

- Politique professionnelle, cf. ci-avant

\section{Compétences, motivation et objectifs}

La politique professionnelle était et reste pour moi une priorité. C'est dans ce sens que je souhaite, au terme de ma carrière, mettre mon expérience professionnelle et politique à disposition de la Commission de déontologie de la FMH en me présentant pour un dernier mandat en tant que vice-président. 